\title{
Simulation of nanoparticles interacting with a cell membrane: probing the structural basis and potential biomedical application
}

\author{
Xiao Zhang $\mathbb{1}^{1}$, Guanghui $\mathrm{Ma}^{1,2}$ and Wei Wei $\mathbb{B}^{1,2}$
}

\begin{abstract}
Nanoparticles (NPs), owing to their ultrasmall size, have been extensively researched for potential applications in biomedicine. During their delivery and functionalization within the organism, they frequently interact with cells. The resulting nano-bio interfaces between the NPs and cell membrane play an important role in dominating the physiological effects of NPs. Therefore, understanding how the properties of NPs affect their nano-bio interface interactions with the cell membrane is important. Compared to experimental and theoretical analyses, simulations can provide atomic-level accuracy regarding dynamic changes in structure, which can reveal the mechanisms of nano-bio interface interactions for feasible modulation. Thus, we reviewed the current advances in nano-bio interfaces from the perspective of simulations. This study will determine how the properties of NPs affect their interactions with cell membranes to provide insights for the design of NPs and summarize their corresponding biomedical applications.
\end{abstract}

\section{Introduction}

Nanoparticles (NPs) are typically defined as particles of matter with a diameter between 1 and $100 \mathrm{~nm}$, regardless of their morphological characteristics ${ }^{1}$. Because of this extraordinarily small size, NPs usually exhibit novel physiochemical properties, such as colloidal, optical, and electric properties, which are different from those of bulk materials ${ }^{2}$. NPs have prominent advantages because of their ultrasmall size and high surface-area-to-volume ratio, which are favorable for drug loading, chemical and geometric tunability, penetration and diffusion, and ability to interact with biomolecules. Accordingly, NPs have attracted significant attention for their potential applications in biomedicine ${ }^{3-5}$.

After NPs enter the body, they are transported to tissues and organs to exert their physiological effects. During

\footnotetext{
Correspondence: Wei Wei (weiwei@ipe.ac.cn)

${ }^{1}$ State Key Laboratory of Biochemical Engineering, Institute of Process Engineering, Chinese Academy of Sciences, No. 1 Bei-Er-Tiao, Zhong-GuanCun, Haidian District, 100190 Beijing, People's Republic of China

${ }^{2}$ School of Chemical Engineering, University of Chinese Academy of Sciences, No. 19A Yuquan Road, 100049 Beijing, People's Republic of China
}

their delivery and functionalization within the organism, they frequently interact with cells, inducing a series of nano-bio interface interactions ${ }^{6}$. These nano-bio interfaces between NPs and cell membranes play an important role in the physiological effects of NPs. Understanding how the properties of NPs affect their nano-bio interface interactions with the cell membrane is essential for biomedical applications ${ }^{7}$, and many studies have focused on this aspect. For example, Donahue et $\mathrm{al}^{8}{ }^{8}$ described three distinct aspects of NP-cell interactions: (i) NP cellular uptake; (ii) NP intracellular trafficking; and (iii) underlying kinetics of these cellular and subcellular nano-bio interactions. Gagner et al. ${ }^{9}$ provided an advanced understanding of the factors influencing nano-bio interactions (e.g., NP morphology, biomolecule placement, and orientation) and corresponding strategies to manipulate nano-bio interactions for nanomedicine design. Regardless of the experimental methods, there are always limitations on the detection precision of reflecting the dynamic interaction process of the nano-bio interface, mainly due to the extraordinarily small size of NPs. This limits our understanding of nano-bio interface 
interactions and the potential de novo design of new NPs for application in biomedicine.

Simulation is an approximation of the operation of a process or system, which can provide atomic-level accuracy to reveal the underlying mechanisms of physiological phenomena ${ }^{10}$. It is an ideal approach to study nano-bio interface interactions, revealing the dynamic endocytosis process between NPs and cell membranes. Based on different research purposes, diverse simulation methods have been developed and applied, such as all-atom molecular dynamics (AAMD), coarse-grained molecular dynamics (CGMD), and dissipative particle dynamics $(\mathrm{DPD})^{11,12}$. However, a general limitation is that only one or a few physical properties of NPs are focused upon in one work, which cannot comprehensively represent the overall characteristics of NPs. In addition, there is no systematic summary of how simulations can be applied in the biomedical field. These factors greatly limit the development of NPs and their potential applications in biomedicine. Thus, this review will summarize the progress in current simulation methods for visualizing nanobio interface interactions, which will cover most of the physical properties of NPs and their corresponding surface modifications based on grafted chemistries, and practical biomedical applications. The goal of this review is to determine, via simulation, how the properties of NPs affect their interaction with cell membranes, to guide the rational de novo design of NPs, and expand their potential biomedical applications.

\section{Simulation methods applied in nano-bio interface interactions}

To examine the nano-bio interface interactions, the construction of the initial model and choice of the simulation method are important. Considering the apparent size difference between the NP and cell at the interface, the structure of the NP is usually represented according to its actual situation, while the cell is usually simplified as a planar cell membrane. Because the cell membrane is mainly composed of lipids and proteins, the current models can be classified into three types: models with a single lipid component, with multiple lipid components, and with a single lipid component combined with a membrane protein. To reduce structural complexity and improve computational efficiency, the cell membrane is in practice usually constituted by the most common phosphatidylcholine ${ }^{13}$, unless specific lipid components or membrane proteins need to be studied.

The ideal models for NP and the cell membrane are allatom models, wherein the NP and cell membrane can be constructed in terms of their atomic composition, and the bonding information between atoms can be directly obtained from the force field parameters (based on quantum mechanics) of the AAMD simulation method ${ }^{14}$.
Taking a 1-palmitoyl-2-oleoyl-sn-glycero-3-phosphocholine (POPC) lipid molecule as an example, its initial structure consists of multiple atoms, and the covalent and noncovalent interaction parameters between atoms can be determined using the CHARMM force field (Fig. 1a) ${ }^{15}$. These all-atom models and methods can benefit studies on atomic precision; however, their computational efficiencies and computing resources need to be addressed. This simulation method is suitable for NPs and cell membranes with simple structures (such as onedimensional carbon nanotubes, two-dimensional graphene, etc.) and compositions (such as single lipid composition, multiple lipid composition, and a simple membrane protein), respectively.

For sophisticated systems, all-atom models are limited due to their expensive calculation costs and inability to simulate physiological phenomena on a real-time scale. Hence, advanced models and matching simulation methods are imperative. Therefore, coarse-grained (CG) models have been developed to simplify all-atom models. In CG models, molecules are not represented by individual atoms but by "pseudoatoms" representing groups of atoms, that is, in the form of beads. By significantly decreasing the degree of freedom of the system, a longer simulation period can be studied in line with physiological phenomena; however, it comes at the expense of molecular detail. According to the CG degree of the model and

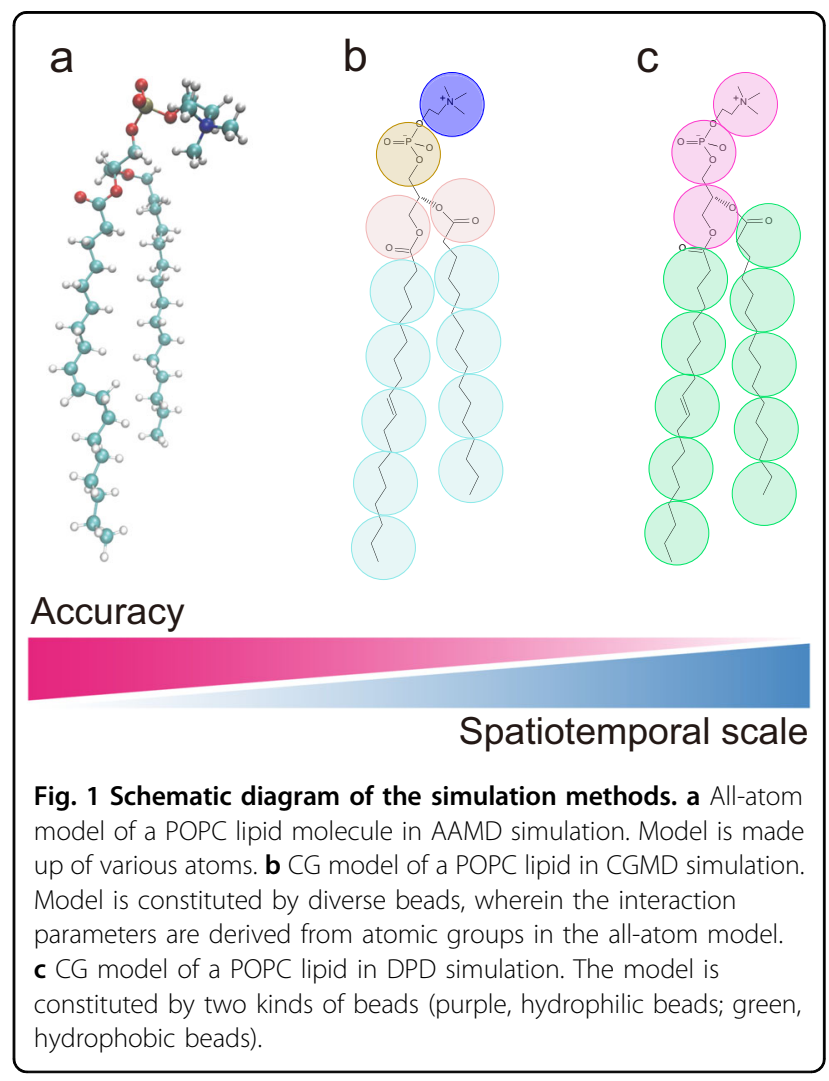


definition of the interactional parameters between beads, CG simulation methods mainly include CGMD and DPD. The CGMD simulation method is a direct simplification of the AAMD simulation because it uses a single bead to represent a group composed of several atoms with similar properties ${ }^{16}$. The interaction parameters between the beads are also converted and determined from the AAMD force field, which ensures the consistency of the simulation results. As shown in Fig. 1b, the all-atom POPC model is simplified using 13 beads, which is based on the Martini CG force field ${ }^{17}$. Each bead is converted from an atom group and represents the nature of its group, such as the polar hydrophilic and hydrophobic beads in the head and tail, respectively. Their interactional parameters are also determined in the Martini CG force field.

DPD is a stochastic simulation technique for simulating the dynamic and rheological properties of simple and complex fluids with greater simplifications ${ }^{12}$. The interaction parameters between the beads are artificially defined, but it is necessary to ensure that the macroscopic properties of the model (e.g., electric charge density and diffusion coefficient) are consistent with the all-atom model. As shown in Fig. 1c, the all-atom POPC model is also simplified using beads, but each bead has only two attributes, that is, hydrophilicity and hydrophobicity. The interactional parameters are empirically set, which requires further data for verification. Therefore, using CG simulations, NPs and cell membranes can be represented using beads, and the nano-bio interface interactions can be examined on a spatiotemporal scale that is closer to physiological phenomena.

After constructing the initial structure and selecting the appropriate force field, the dynamic process of the nanobio interface interaction can be simulated and reproduced for detailed investigation based on the corresponding theoretical principle. All of the AAMD, CGMD, and DPD simulation methods are based on Newton's second law, following the equation $F=m a$. The mass of each component unit (atoms/beads) in the NPs is determined in the corresponding force field, the initial coordinate is governed by the initial model, and the initial velocity is allocated with an appropriate Maxwell-Boltzman distribution for the specified temperature. Thus, the main differences of these simulation methods are concentrated on the expression of parameter $F$.

In the AAMD simulation method, the parameter $F$ is determined by a specific potential energy function $U$ (which varies slightly in different force fields), following the equation $F=-\nabla U$. The functional equation of $U$ is usually composed of two aspects: covalent interactions and noncovalent interactions. Taking the CHARMM force field as an example, the covalent interactions include the bond $\left(U_{\text {bond }}\right)$, angle ( $\left.U_{\text {angle }}\right)$, dihedral ( $\left.U_{\text {dihedral }}\right)$, and improper $\left(U_{\text {improper }}\right)$ interaction terms, and the noncovalent interactions include the van der Waals $(\mathrm{VdW})\left(U_{\mathrm{VdW}}\right)$ and electrostatic $\left(U_{\text {elec }}\right)$ interaction terms, that is, $\quad U=U_{\text {bond }}+U_{\text {angle }}+U_{\text {dihedral }}+U_{\text {improper }}+$ $U_{\mathrm{VdW}}+U_{\text {elec }}$. Each term is a function of the atomic coordinates or charge, with its interior constant coefficients assigned in the force fields. To avoid endless calculation and improve efficiency, the cutoff distance is utilized for the noncovalent interactions. Based on the above conditions, the coordinate and velocity of each atom at any time can be determined in the simulation process. The integrated trajectory data can directly display the dynamic process of nano-bio interface interactions, which can be further analyzed to reveal the underlying mechanism.

In the CGMD simulation method, the source of parameter $F$ is similar to that in the AAMD simulation method. As a consequence of representing multiple atoms with a bead, the constant coefficients in each term change accordingly. The properties and constant coefficients of beads are also determined in the force fields, which are optimized to allow more realistic and accurate simulations of molecular systems. For example, a newly published study updated the most commonly used Martini force field ${ }^{18}$. The number of bead types was substantially increased to a total of 843 bead types that correspond to $\sim 15$ times more options than in the previous version of Martini, and the corresponding constant coefficients were optimized. This updated CGMD model will greatly promote the development of materials science and highthroughput drug design.

In the DPD simulation method, there is a clear difference from the above two methods in the expression of parameter $F$. To retain the characteristics of Brownian motion in the system, beads $i$ and $j$ interact through a simple pairwise force consisting of a conservative force $\left(F_{i j}^{C}\right)$, a dissipative force $\left(F_{i j}^{D}\right)$, and a random force $\left(F_{i j}^{R}\right)$. Therefore, the total force applied on each bead $i$ due to bead $j$ is given as a sum of these three terms, in the equation $F=F_{i j}^{C}+F_{i j}^{D}+F_{i j}^{R}$. Note that the conservative force, $F_{i j}^{C}$, is a soft repulsion potential of beads, which allows much greater length and time scales in DPD. Under this special potential, DPD beads may overlap with each other in simulations; thus, special boundary conditions or modified potential functions are necessary in practice. The dissipative force, $F_{i j}^{D}$, represents the effects of viscosity, and it depends on both the relative positions and the velocities of beads. The random force, $F_{i j}^{R}$, represents the effects of thermal fluctuations, which also depend on the relative positions of the beads.

Overall, the basic theoretical principles of AAMD, CGMD, and DPD simulation methods are consistent (following Newton's second law), but there are differences in the specific expression of interior parameters, especially $F$. The CG degree of the component unit and appropriate 
force fields in each simulation method exhibit obvious differences, along with the accuracy of the models and the spatiotemporal scale of the simulations. The comparison of AAMD, CGMD, and DPD simulation methods is summarized in Table 1, and a more detailed and mathematical introduction can be found in a previous review ${ }^{19}$.

\section{Probing nano-bio interface interactions between NPs and the cell membrane}

Using the above simulation methods, nano-bio interface interactions can be systemically studied to clarify their underlying mechanisms. Considering that the majority of nano-bio interfaces occur during cellular uptake, which can be influenced directly by the physicochemical properties of NPs, various studies have focused on the size, shape, elasticity, charge, hydrophobicity, and surface modification of NPs (Fig. 2) ${ }^{19,23,24}$. The current progress and conclusions are comprehensively summarized as follows.

\section{Size}

The size of NPs can directly affect their nano-bio interface interaction. Therefore, determining the appropriate size of NPs to achieve a high internalization rate and small interference with the cell membrane can provide clues for experiments, which can improve endocytosis efficiency and reduce production costs. Considering the structural complexity of NPs, the all-atom model is unable to achieve this because of its spatiotemporal limitations. Thus, CG models and corresponding simulation methods are generally utilized. Huang et ll. $^{25}$ used a CGMD model to simulate the receptor-mediated endocytosis of NPs of different sizes and shapes. To ensure physiological rationality and improve computational efficiency, the receptors and their corresponding ligands were simplified using beads on the cell membrane and surface of the NPs, respectively (Fig. 3a). They found that the size of the NPs determined whether endocytosis could be achieved. Meanwhile, the shape of the NPs broke the symmetry of the curvature energy landscape and hence dictated the endocytic pathways. Specifically, at an intermediate NP size $(R=7.5 \sigma, \sigma \sim 2 \mathrm{~nm})$, endocytosis was faster than that of the other NP sizes $(R=5.0 \sigma / 10.0 \sigma /$ $12.5 \sigma$ ) (Fig. 3b). By comparison, Gupta et al. ${ }^{26}$ also conducted CGMD simulations to explore the permeation of dodecanethiol-coated neutral hydrophobic gold NPs (AuNPs) of different sizes $(2-5 \mathrm{~nm})$ through a model skin lipid membrane. The $2 \mathrm{~nm}$ AuNPs did not induce significant structural changes in the bilayer, while larger AuNPs $(3-5 \mathrm{~nm})$ significantly changed the structure and packing. The free energy of permeation and diffusivity indicated that the $2 \mathrm{~nm}$ AuNPs achieved maximum

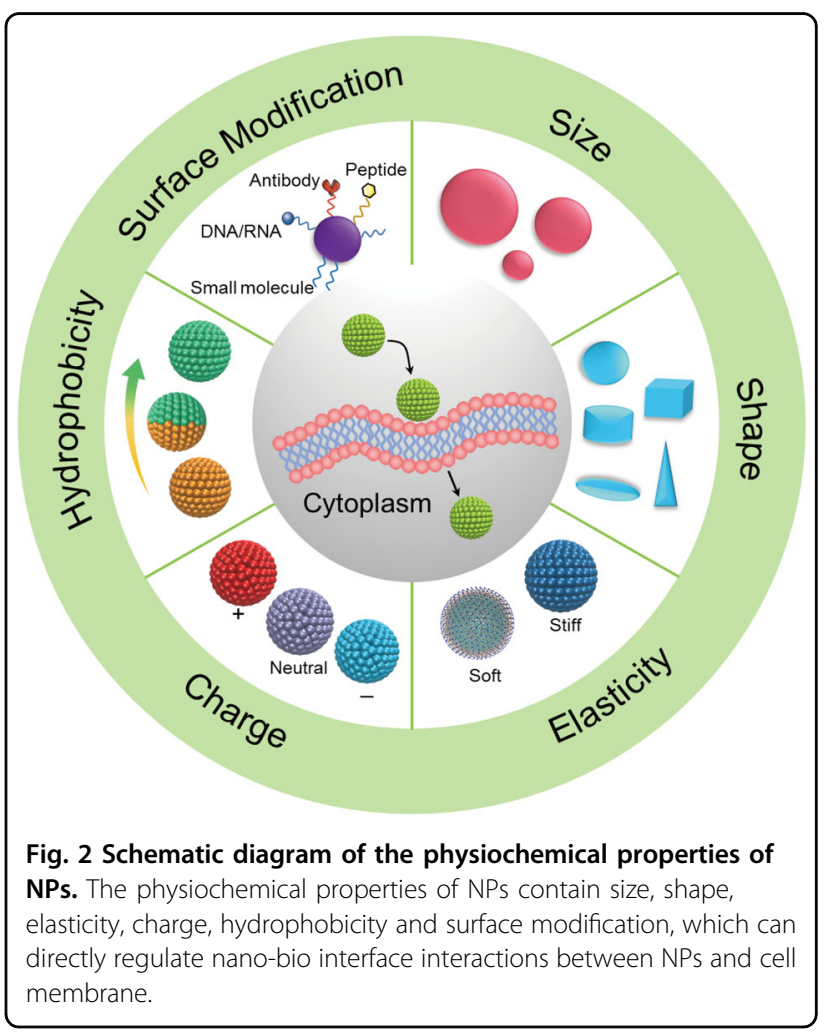

Table 1 Comparison of AAMD, CGMD and DPD simulation methods.

\begin{tabular}{|c|c|c|c|c|c|}
\hline Method & Component unit & Spatial scale (m) & Time scale (s) & Characteristics & Force fields \\
\hline AAMD & Atom & $10^{-9}$ & $10^{-9}$ & $\begin{array}{l}\text { Explicit molecular details at atomic level } \\
\text { Limited spatiotemporal scale }\end{array}$ & $\begin{array}{l}\text { CHARMM }^{15} \\
\text { GROMOS }^{20} \\
\text { OPLS } \\
\text { AMBER }^{22}\end{array}$ \\
\hline CGMD & Bead & $10^{-8}$ & $10^{-7}$ & $\begin{array}{l}\text { Enhanced spatiotemporal scale compared with AAMD } \\
\text { Coarse information at atomic level }\end{array}$ & Martini ${ }^{17,18}$ \\
\hline DPD & Bead & $10^{-7}$ & $10^{-5}$ & $\begin{array}{l}\text { Retained characteristics of Brownian motion } \\
\text { Potentially overlapped beads in space }\end{array}$ & DPD potentials \\
\hline
\end{tabular}




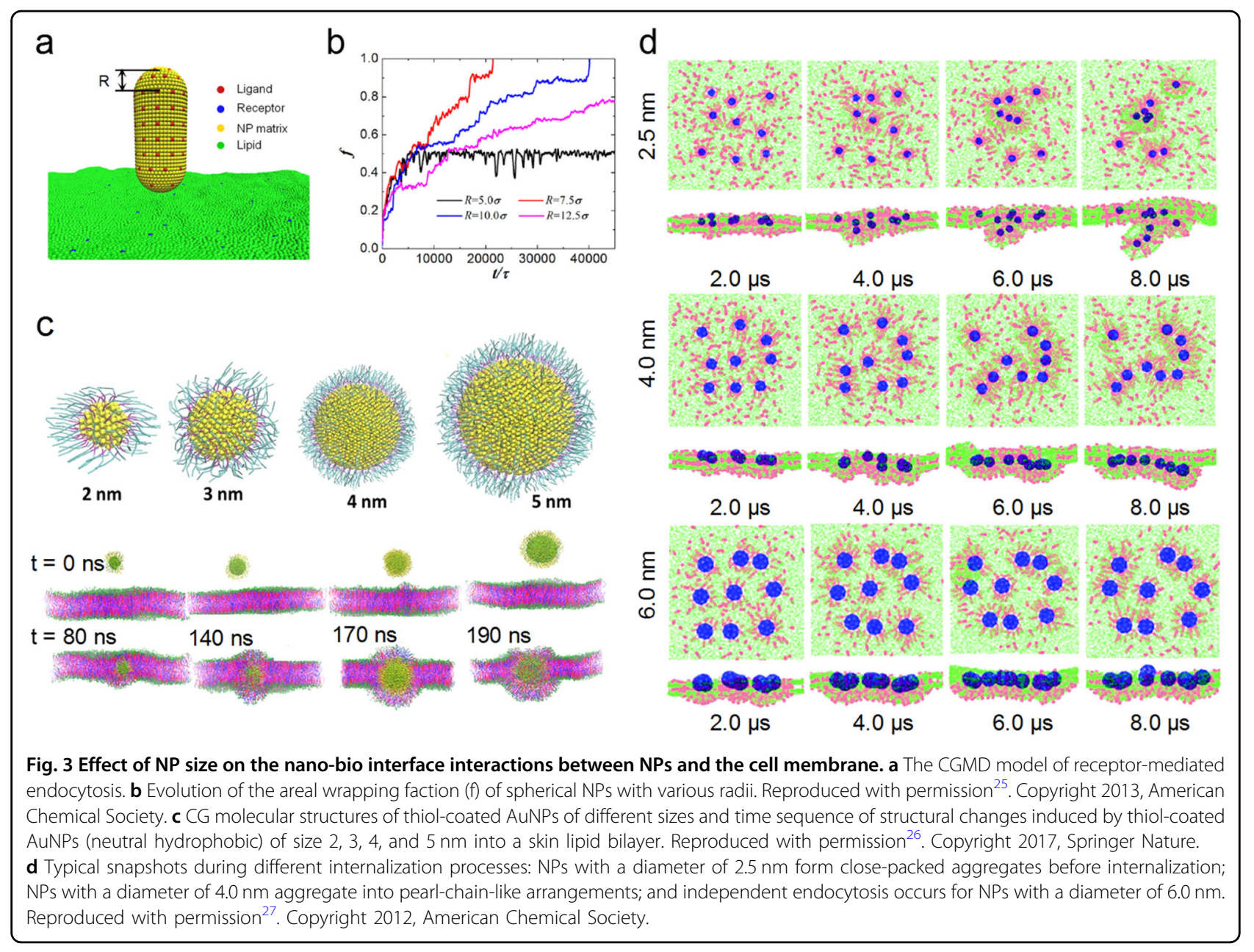

permeability. This result appeared to be contradictory to the previous result (Fig. 3c) ${ }^{25}$. On the one hand, this may be attributed to the specific modification of dodecanethiol, which endowed the small NPs with the property of readier permeation. On the other hand, this may have resulted from the ultrasmall particle size $(\leq 5 \mathrm{~nm}) \mathrm{com}$ pared to the larger particle size $(\geq 10 \mathrm{~nm})$. This suggested that for the special size range of NPs $(1-100 \mathrm{~nm})$, slight changes in size may affect their nano-bio interface effects.

Considering that different NPs exist synchronously at the nano-bio interface, the coupling effect among these NPs is a non-negligible factor. Thus, in addition to the size effect of a single NP, the coupling effect of multiple NPs of different sizes was investigated. Yue et al. ${ }^{27}$ used a particular variant of DPD simulations to detect the endocytosis of multiple NPs using a lipid membrane. They found that the endocytosis of multiple NPs was a size-dependent cooperative process. That is, smaller NPs with sizes of $2.5 \mathrm{~nm}$ generally clustered into close-packed aggregates, NPs with intermediate sizes of $4.0 \mathrm{~nm}$ tended to aggregate into a linear pearl-chain-like arrangement, and larger NPs with sizes of $6.0 \mathrm{~nm}$ were apt to separate from each other and be internalized independently (Fig. 3d). Furthermore, in contrast to a single NP, the energy cost associated with the strong local membrane deformation in wrapping could be substantially reduced by multiple NP clustering, which facilitated the wrapping process.

Most of the NPs are represented by beads that are stacked in a spherical or quasi-spherical structure, but this is not suitable for NPs with unique structures. Guo et al. ${ }^{28}$ expanded the NP scope to an emerging two-dimensional (2D) graphene nanosheet (GN). They discovered the permeation of a small GN (side length $=3.5 \mathrm{~nm}$ ) into the bilayer center through insertion and rotation driven by transbilayer lateral pressure. For large GNs (side length = $7 \mathrm{~nm}$ ), the translocation underwent a vesiculation process driven by complicated energetic contributions. These results provide fundamental insights into the cellular internalization mechanisms of graphene-based NPs and enrich the understanding of the size effects of NPs with different dimensions. In summary, the cellular uptake rate and internalization behavior of $\mathrm{NP}(\mathrm{s})$ can vary greatly based on their size, and the optimal size is dependent on 
the other properties of NPs (such as surface modification, aggregation degree, and dimension).

\section{Shape}

With the development of nanotechnology, NPs can be designed and made into various shapes that are in demand, such as spheres, ellipsoids, rods, and cones. When NPs with different shapes interact with cells, their nano-bio interface interactions clearly exhibit different properties. Yang et al. ${ }^{29}$ used DPD simulation methods to investigate the physical translocation processes of NPs with different shapes (e.g., spheres, ellipsoids, rods, discs, and pushpin-like particles) across a lipid bilayer. The penetrating capability of an NP across a lipid bilayer is determined based on the contact area between the particle and lipid bilayer and the local curvature of the particle at the contact point. For a more physiological representation, the cell membrane can be developed from a single lipid component to a multicomponent lipid bilayer model (Fig. 4a). The efficacy of NP penetration across this multicomponent lipid layer was rod > disc > sphere (Fig. $4 \mathrm{~b})^{30}$, which was also consistent with the above results.

In addition to bare NPs with different shapes, the receptor-mediated endocytosis of NPs with different shapes was also explored. Through the specific definition of the interaction parameters for receptor-ligand interactions, the nano-bio interface can be studied more intensively. Shen et al. ${ }^{31}$ developed CGMD models for NPs to systematically investigate the receptor-mediated membrane wrapping of NPs with different shapes. Consistent with the above results ${ }^{25}$, the receptors and their corresponding ligands were simplified using beads on the cell membrane and surface of NPs, respectively (Fig. 4c). The membrane wrapping efficiency of NPs during endocytosis was a result of the competition between the receptor diffusion kinetics and thermodynamic driving force. The receptor diffusion kinetics are referred to as the kinetics of receptor recruitment and are affected by the contact edge length between the NP and membrane. The thermodynamic driving force represents the amount of free energy required to drive NPs into a cell. For example, oblate NPs needed to overcome a larger energy barrier than prolate NPs. However, the oblate NP had a significantly larger contact edge length, which enabled it to be fully wrapped faster than the prolate NP (Fig. 4d). Thus, ligand-modified NPs have advantages and disadvantages in endocytosis, which need to be balanced when applied practically. In line with this idea, Vacha et al. $^{32}$ focused on passive endocytosis across lipid membranes and performed CGMD simulations of phospholipids and NPs with various shapes. They found that the efficiency of passive endocytosis was higher for spherocylindrical NPs than for spheres and that endocytosis was suppressed for NPs with sharp edges.

More detailed endocytosis kinetics were further clarified for ligand-coated NPs with different shapes, such as spherical-, rod-, and disc-shaped NPs ${ }^{33}$. Depending on the shape anisotropy of NPs, their internalization processes can be divided into the invagination and wrapping stages. As a result of the strong ligand-receptor binding energy, the membrane invagination stage was characterized by the rotation of NPs to maximize their contact area with the membrane (Fig. 4e). The kinetics of the wrapping stage were mainly dominated by the membrane tension and area of the NPs with the largest local mean curvature, where the membrane was most strongly bent (Fig. 4f). Therefore, NPs with various shapes displayed different favorable orientations for the two stages, and one or more orientation rearrangements might be required during the endocytosis process. Overall, these studies showed the effects of varying shapes of NPs on nano-bio interface interactions and established their corresponding theoretical mechanisms.

\section{Elasticity}

The elasticity of NPs is also an important parameter in nano-bio interface interactions. In contrast to size and shape, elasticity is not directly visible on the constructed models. Thus, special methods are needed in the simulation process. Sun et al. ${ }^{34}$ compared poly(lactic-co-glycolic acid) (PLGA)-lipid (P-L) NPs with PLGA-water-lipid (P-W-L) NPs (including liposomes) to understand the mechanism of the rigidity-regulated cellular uptake of NPs. Rigid P-L NPs were fully wrapped by the cell membrane and internalized smoothly. However, the less rigid $\mathrm{P}-\mathrm{W}-\mathrm{L}$ NPs underwent significant deformation during internalization before they were finally trapped within the cell membrane, indicating a more complicated process.

Similarly, elasticity can be artificially modified using the interactional parameter (e.g., bending rigidity) of the beads constituting the NP. Shen et al. ${ }^{31,35}$ modeled the NP using a thin elastic shell with an elasticity that could be systematically changed by tuning its bending rigidity constant (Fig. 5a). Based on this model, the contribution of elasticity to the nano-bio interface interaction was examined. Comparatively, soft spherical NPs had significantly higher energy barriers due to their ability to deform. Owing to the increased energy barriers, soft spherical NPs needed to recruit more receptors to provide the driving force for membrane wrapping. Therefore, they were not fully wrapped compared to the rigid NPs (Fig. 5b). Moreover, Shen et al. ${ }^{36}$ further investigated the polyethylene glycol modification of NPs with different elasticities on the membrane wrapping processes (Fig. 5c). The PEGylated liposomes were partially wrapped, while 


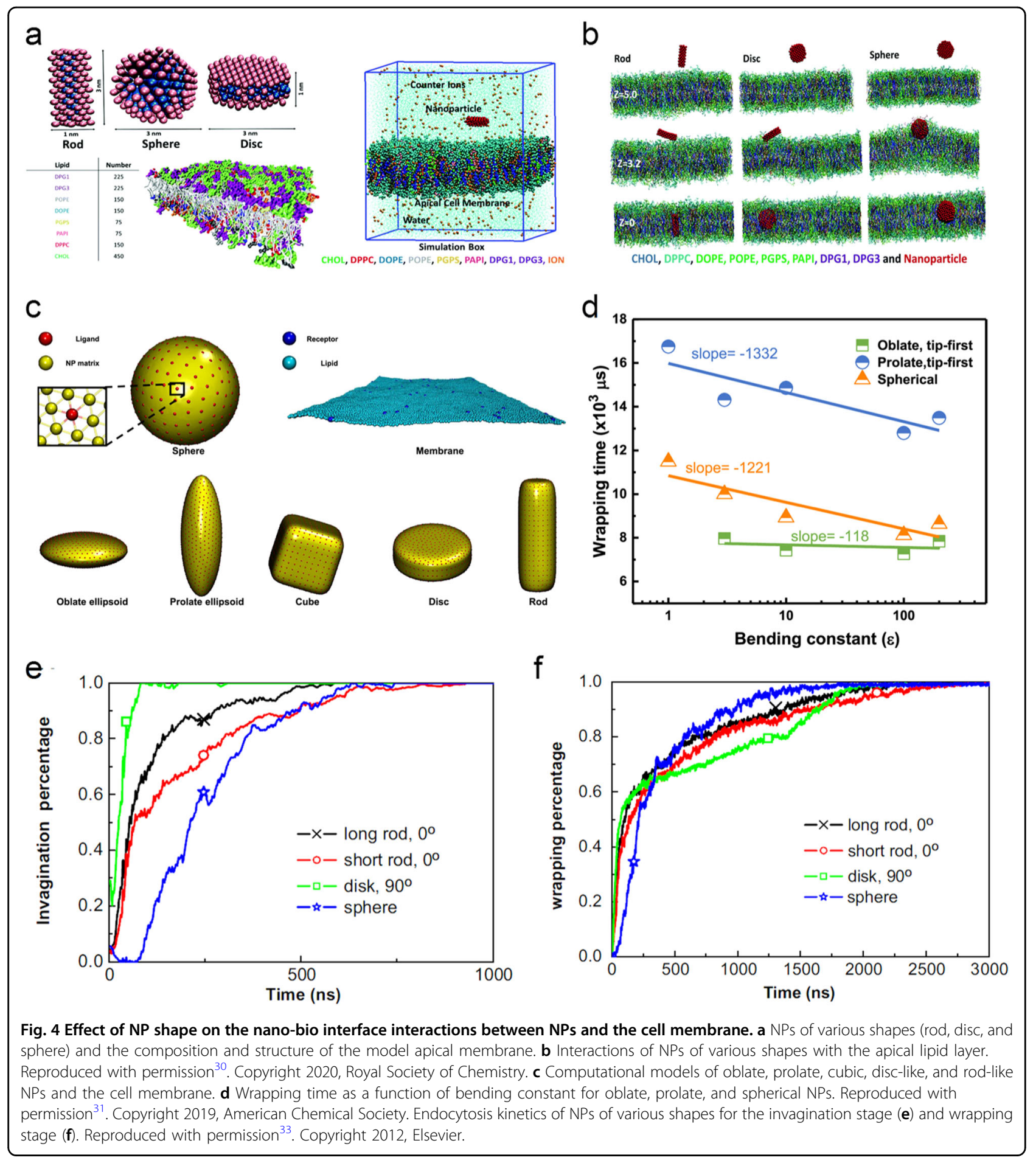

the PEGylated rigid NPs were fully wrapped by the cell membrane. They concluded that the main reasons were the large free energy penalty induced by PEG aggregation and ligand-free regions on the liposome surface (Fig. 5d). Accordingly, these studies provided fundamental insights into the endocytosis of NPs with different elasticities; that is, compared to soft NPs, rigid NPs tend to be transported across the membrane, which can help in designing NPs with high efficiencies for potential applications.

\section{Charge}

Considering the natural negative charges of cell membranes, the charge of NPs can be modified to regulate their nano-bio interface interactions with the cell 


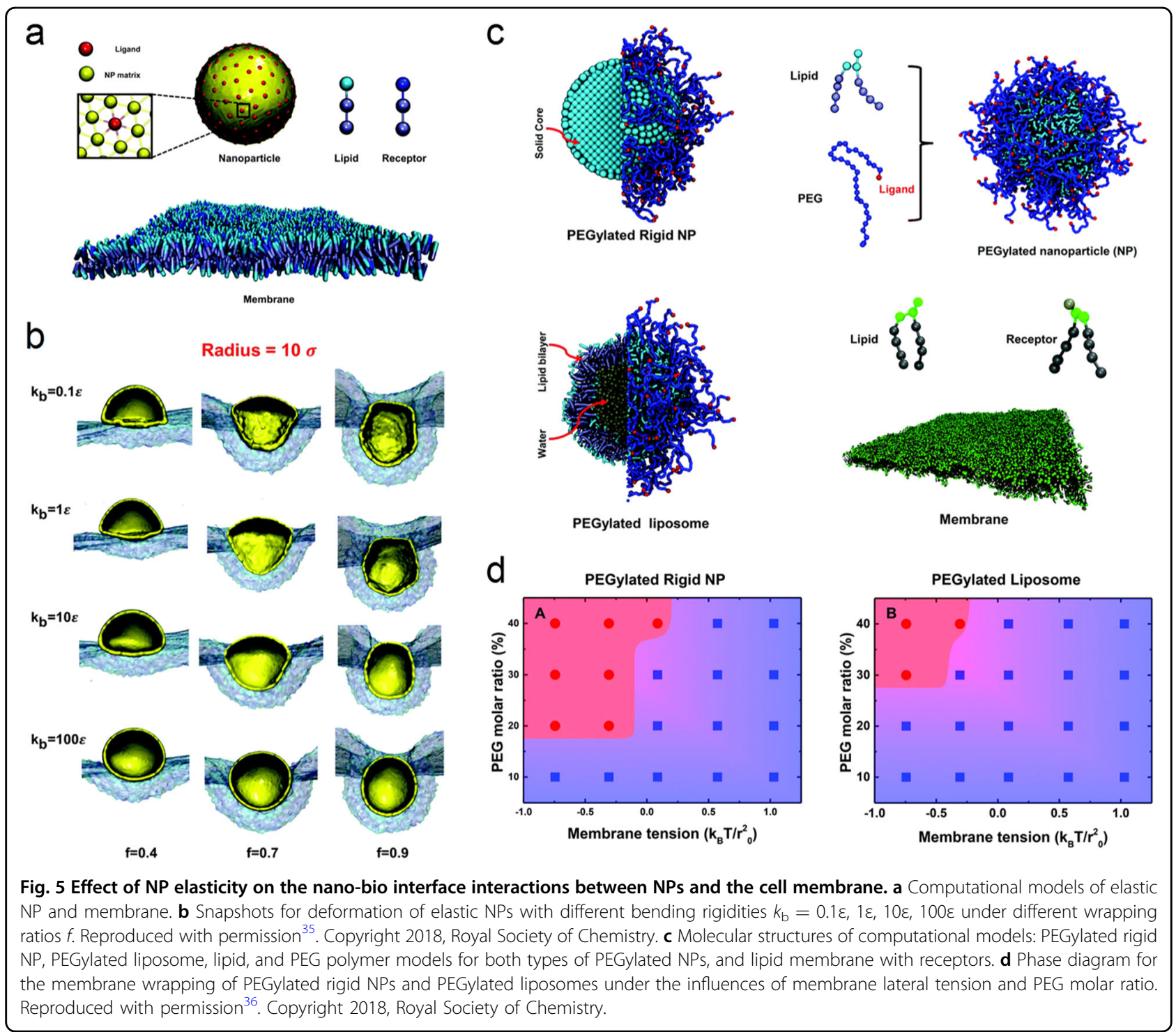

membrane. Accordingly, Nangia et al. ${ }^{37}$ developed CGMD simulations capable of providing quantitative predictions of experimentally measurable rate constants and half-lives to characterize the effect of NP surface charge on translocation through negatively charged cell membranes. As expected, NPs with negatively charged surface functionalization were electrostatically repelled from the cell membrane, while neutral and positive NPs could interact with the cell membrane. Furthermore, the surface charge density of neutral and positive NPs played a key role in their translocation processes: asymmetrically charged NPs undergo an electrostatics-driven reorientation in the vicinity of the bilayer, thereby enhancing the $\mathrm{NP}$-membrane contact area and disrupting the selfassembly of the membrane lipids (Fig. 6a, b).

However, in many other studies, the cell membrane was constructed from neutrally charged lipids (e.g., DPPC,
POPC), which may mediate different nano-bio interfacial interactions. The Gu group ${ }^{38,39}$ investigated the interactions between NPs with different charge properties and neutral membrane bilayers (Fig. 6c). The adsorption behavior of NPs was largely dominated by the surface charge properties. The higher surface charge density induced better adsorption and thus relative local membrane thinning. Meanwhile, the variation in the adsorption behavior of NPs with the charge density was quite different for positively and negatively charged NPs. For positively charged NPs, the adsorption became dramatically weaker with decreasing charge density. However, the adsorption of negatively charged NPs weakened more gently than that of positively charged NPs. In general, negatively charged NPs tended to adsorb onto the lipid bilayer more easily (Fig. 6d). This difference for positively and negatively charged NPs is mainly induced by the 


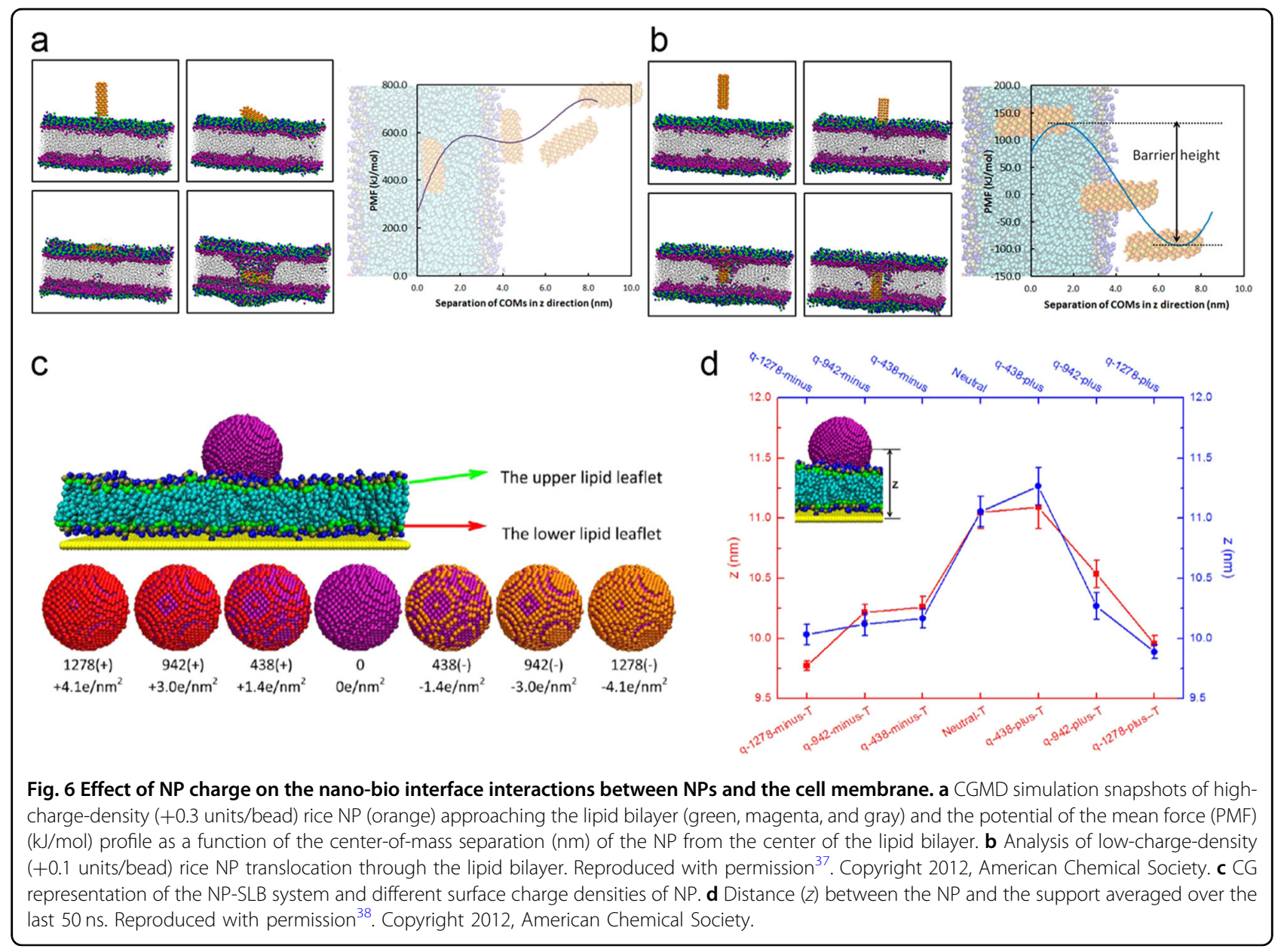

dipole layer formed by charged head groups of lipid molecules with a positively charged group outside. Thus, the choice of lipid type in the simulation is very important for the final outcomes and needs to be selectively determined according to the actual problems.

\section{Hydrophobicity}

Hydrophobicity is the physical property of a molecule that allows it to be repelled from a mass of water. Hydrophobic molecules tend to be nonpolar; thus, they prefer neutral molecules and nonpolar solvents. Because water molecules are polar, hydrophobic molecules do not dissolve well among them. Hence, corresponding functionalizations can be adopted to mediate molecular hydrophobicities, expanding their practical applications. For example, hydrophobic graphene was oxidized using a modified Hummers method $^{40}$ to improve its hydrophilicity for potential biomedical applications. The cell membrane is composed of abundant amphiphilic lipids, with hydrophilic heads and hydrophobic tails. Accordingly, nano-bio interface interactions are inevitably influenced by the hydrophobicity of NPs.
Theoretically, the hydrophobic effect of an NP is a result of the interplay of all component beads: for hydrophilic beads, the cell membrane acts as a potential barrier, making beads tend to concentrate in the hydrophilic head regions of the bilayer membrane; for hydrophobic beads, the cell membrane acts as a potential trap, confining beads in the hydrophobic interior region of the bilayer membrane. Therefore, the proportion and arrangement of hydrophilic/hydrophobic beads will affect the free energy landscape, which has been investigated using simulation. For example, Gupta et al. ${ }^{41}$ constructed a series of NPs with hydrophobic and hydrophilic beads to mimic the permeation of NPs through the skin lipid bilayer (Fig. 7a). NPs with a homogeneous 2:1 ratio of hydrophobic to hydrophilic content were found to be more effective in permeation alone and with protein, which demonstrated that appropriate hydrophobicity can promote the endocytosis of NPs (Fig. 7b). Similarly, Su et al. ${ }^{42}$ studied the passive translocation of NPs with a size of approximately $1 \mathrm{~nm}$ and tunable degrees of hydrophobicity through lipid bilayer membranes (Fig. 7c). The results displayed a narrow window of high translocation rates for NPs with a 


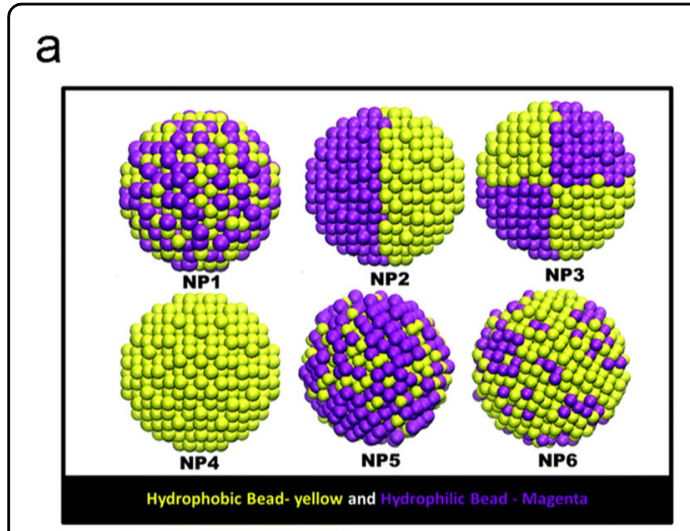

C

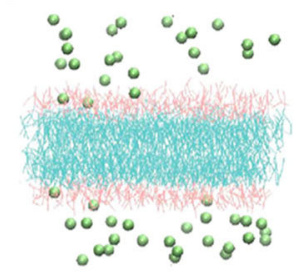

$\mathrm{H}=0$

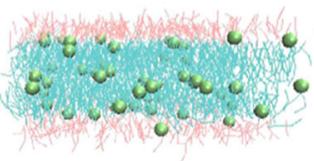

$\mathrm{H}=0.8$

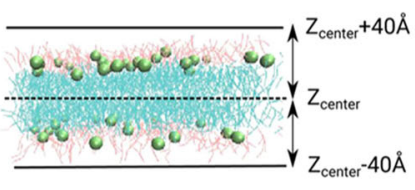

$\mathrm{H}=0.5$

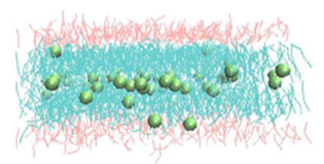

$\mathrm{H}=1$ b
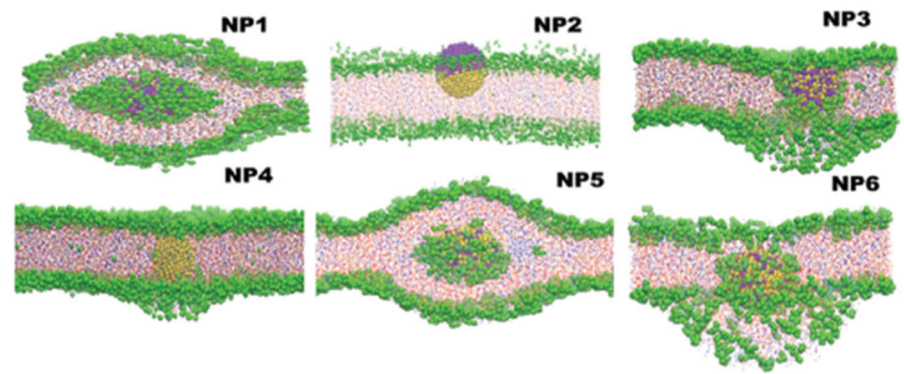

CER -Red, CHOL-Orange, FFA-Blue, Hydrophobic Bead-yellow,
Hydrophilic Bead-Magenta and Head Group-Green

d

Fig. 7 Effect of NP hydrophobicity on the nano-bio interface interactions between NPs and the cell membrane. a Structure of different NPs. The hydrophobic and hydrophilic beads are represented by yellow and magenta, respectively. b Final snapshot of each NP and skin lipid bilayer system at the end of a 6 us unconstrained simulation. Reproduced with permission ${ }^{41}$. Copyright 2018, Royal Society of Chemistry. c Snapshots: lipid membranes interacting with 50 NPs at different values of hydrophobicity $(H=0,0.5,0.8,1)$. $\mathbf{d}$ Free energy profiles as a function of the distance from the membrane center, in the presence of 50 NPs, and at different hydrophobicities, $\mathrm{H}$. Reproduced with permission ${ }^{42}$. Copyright 2017 , American Chemical Society.

balanced hydrophobicity close to $H=0.5$. At this point, the free energy landscape was maximally flat, and the remaining barrier could be associated with the entropy reduction of the tails by accommodating the particle (Fig. 7d). Therefore, NPs with moderate hydrophobicities exhibited the best cellular uptake properties.

\section{Surface modification}

In addition to the above physical properties, NPs can also be influenced by chemical modification according to specific requirements for regulating nano-bio interface interactions. Various molecules have been adopted for the surface modification of NPs, such as chemical molecules, functional peptides, proteins, and nucleic acids ${ }^{43-45}$. Considering the complexity of the system and the efficiency of the simulations, these molecules are generally simplified as beads in actual simulation studies. For example, Shen et al. ${ }^{46}$ systematically investigated the stability and cellular interaction of $\mathrm{pH}$-responsive monolayer- protected AuNPs using CGMD simulations. The AuNPs were codecorated with positively charged $N, N, N$-trimethyl (11-mercaptoundecyl) ammonium (TMA) and 11mercaptoundecanoic acid (MUA) ligands with $\mathrm{p} K_{\mathrm{a}}=$ 5.08 (Fig. 8a). The penetration of AuNPs led to a dramatic increase in free energy because of the deformation of the ligands and hydrophilic functional end groups (Fig. 8b-d). Similarly, Lehn et al. ${ }^{47}$ prepared AuNPs with a monolayer composed of 11-mercaptoundecane sulfonate and octanethiol ligands with three surface morphologies (Fig. 8e, f). They found that increasing the relative proportion of hydrophilic ligands inhibited insertion by imposing a barrier to ligand bending due to the bulkiness of the sulfonate end groups and increased electrostatic repulsion. Finally, AuNP monolayers with additional hydrophobic ligands had larger amounts of exposed hydrophobic surface area in the baseline state, which was shielded in the bilayer, leading to greater hydrophobic driving forces (Fig. 8g). Moreover, Bai et al. ${ }^{48}$ used a DPD simulation to 


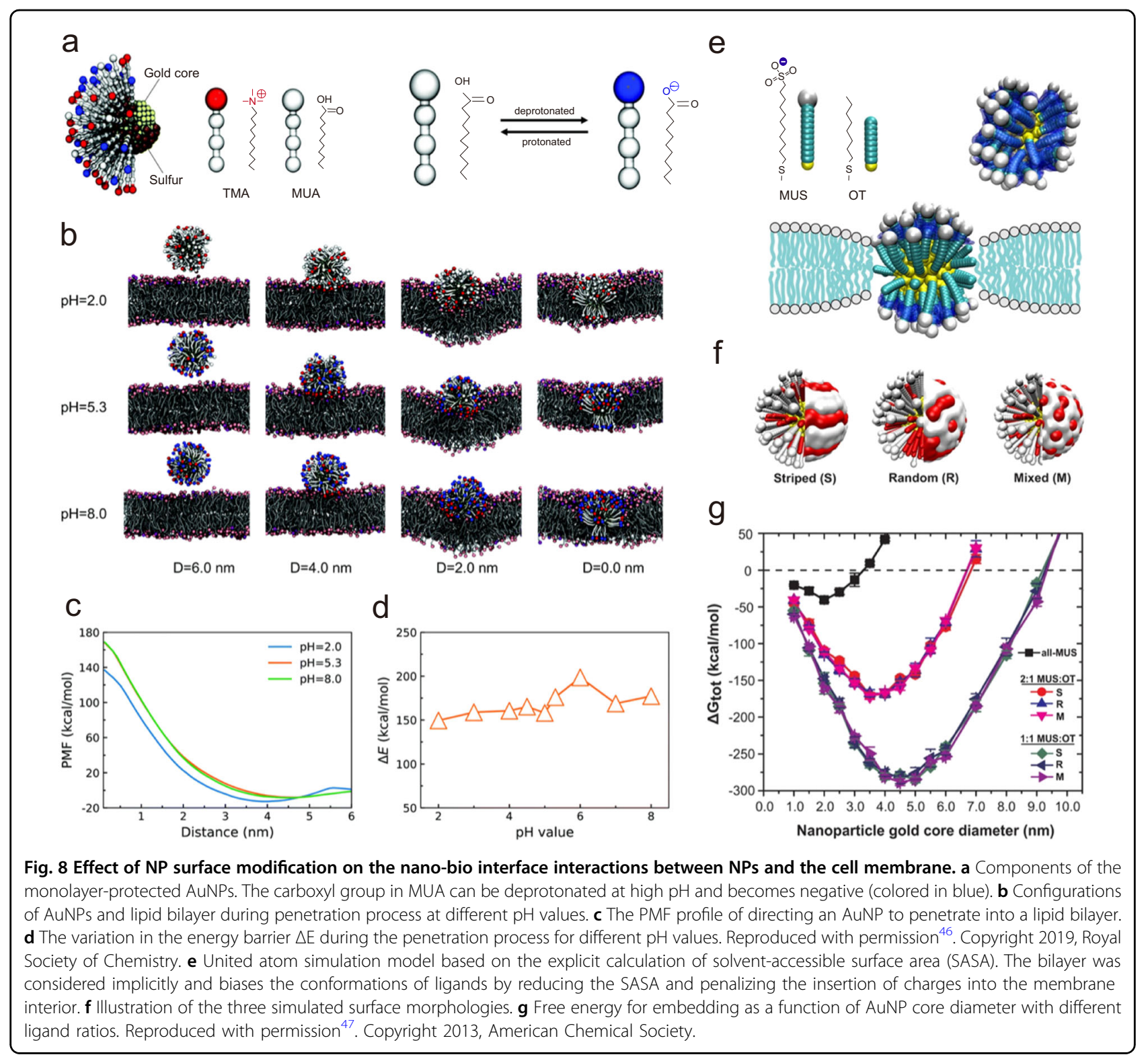

investigate how the physicochemical properties of the coating pulmonary surfactant lipids and proteins affect the membrane response of inhaled NPs. They found that hydrophobic surfactant proteins can promote the uptake of NPs owing to their extra attraction to membrane lipids. However, the engulfment efficiency mainly relied on the density of the coating lipids because of their overwhelming coverage ratio. These results indicated that through surface modification of NPs, the effects of nano-bio interface interactions between NPs and the cell membrane can be regulated and controlled.

Overall, the nano-bio interface interactions between the NPs and cell membrane are related to the physiochemical properties of NPs. Through different simulation methods, a microscopic process of the interface interaction can be explored in detail. Although many studies have involved as many factors as possible and established corresponding theoretical models ${ }^{19,24,49-51}$, it is still difficult to screen an optimal condition for the cellular uptake of NPs because the physiochemical properties are interrelated. However, a plausible rule that is beneficial for the cellular uptake of NPs can be derived. For the size and shape factors, the penetrating capability of an NP across a lipid bilayer is determined mainly based on the contact area between the particle and lipid bilayer and the local curvature of the particle at the contact point; however, the addition of receptor-ligand interactions will make this process more complicated. Rigid NPs could accelerate penetration, and a higher surface charge density may induce better adsorption. Moderate hydrophobicity is conducive to the cellular 
uptake of NPs. In addition to these physical factors, elaborate surface modifications by specific chemistries could purposefully mediate this nano-bio interface interaction.

\section{Nano-bio interface-based biomedical applications}

Simulations cannot only clarify the microscopic mechanism of nano-bio interface interactions between NPs and the cell membrane but also guide the design of practical applications of NPs in biomedicine. Using simulations instead of experiments can reduce the experimental cost, predict the experimental phenomena and expand the potential applications of NPs. In the following sections, using simulations, we will summarize detailed information on the applications of NPs in the biomedical field, such as drug delivery, nanotoxicology, and immune activation.

\section{Drug delivery}

Drug delivery systems are usually constructed and utilized to comprehensively regulate the spatiotemporal distribution of vaccines and drugs in organisms. The fundamental goal is to deliver the appropriate amount of medicine to the right place at the right time to increase the utilization efficiency of the medicine, improve therapeutic efficacy, reduce costs, and decrease side effects. Considering the ultrasmall size of NPs, they are ideal carriers that can induce the targeted delivery and controlled release of therapeutic agents. To obtain the best effects, NPs are typically modified with ligands (coupled with receptors on the cell membrane) or designed as smart delivery platforms (responding to external stimuli, such as light, heat, and magnetism) in practical applications.

Ligand-functionalized NPs are capable of selectively binding to diseased cell populations, which are attractive for improving the efficacy of NP-based drug and gene therapies. Receptor-mediated transcytosis is a fundamental mechanism for transcellular transport. However, NPs functionalized with high-affinity targeting ligands might exhibit undesired off-target binding to healthy cells or decreased transcytosis efficiency. Therefore, Deng et al. ${ }^{52}$ developed a stochastic model to study the endocytosis and exocytosis of NPs across cell membranes and accounted for both clathrin-mediated and actin-mediated endocytosis for internalization and expulsion, respectively. They showed that NPs must have certain avidities with sufficient ligand densities and ligand-receptor binding affinities to be taken up because an avidity that is too high limits particle release from the cell surface. Furthermore, they found that membrane compression due to actin-induced tension tended to break the ligand-receptor bonds and shrink the fusion pore. Therefore, intermediate tension increased fusion pore expansion and NP release, while high tension prohibited particle release. In summary, the surface density of the ligand on NPs and its binding strength with the receptor on the cell membrane need to be carefully considered for efficient drug delivery.

Recently, NPs have been developed as smart delivery platforms that respond to specific stimuli (such as externally applied electric or magnetic fields). These external stimuli can regulate the motion of NPs and endow new nano-bio interface effects. Experimental studies have reported that by applying an electric field, NPs can directly permeate across the cell membrane without the confinement of NPs in endocytic vesicles. Lin et al. ${ }^{53}$ demonstrated this phenomenon and showed that the membrane resealed itself within a microsecond after translocation, with the transmembrane potential being strongly diminished (Fig. 9a). Furthermore, Shimizu et al. ${ }^{54}$ investigated the effect of an external electric field on the permeation of a cationic AuNP across a phospholipid bilayer. They found that the NP delivery behavior was dependent on the electric field strength: as the electric field intensity increased, the transmembrane pore after the direct permeation of the NP changed from recoverable to persistent. Thus, for a charged NP-based drug delivery system, an appropriate electric field strength can reduce biological damage while maintaining a high delivery rate.

Similarly, the application of magnetically driven superparamagnetic NPs in magnetic response drug delivery carriers has also been investigated. Zhang et al. ${ }^{55}$ performed DPD simulations to analyze the rotation frequencies, sizes, and coating patterns of rotational NPs when they interacted with the vesicle to provide novel designs for drug delivery applications. The translocation time needed for the rotational NPs to enter the vesicle decreased with decreasing rotational frequencies and increased with increasing aspect ratio. Moreover, they added the receptor to the cell membrane model to simulate the physiological situation (Fig. 9b). They found that both the rotational speed of the NPs and ligand-receptor binding strength contributed to the mechanism of NP-induced cell membrane rupture in both the attachment and endocytosis modes (Fig. 9c) ${ }^{56}$. This consolidated the conclusion on the magnetic field-driven motion of NPs. Thus, for a superparamagnetic NP-based drug delivery system, the shape is a dominant factor, and the strength of the external magnetic field should be carefully determined to achieve a suitable rotation speed of NPs.

Most drug delivery systems will be endocytosed into cells to perform their function, but some systems with small sizes or special structures will be incorporated into the cell bilayer membrane. In this case, it is important to clarify their motion mode and whether they can perform drug delivery when they are trapped inside the cell membrane. Graphene oxide (GO) is a typical drug delivery carrier because of its specific planar structure and insertion into the inner region of the cell membrane 


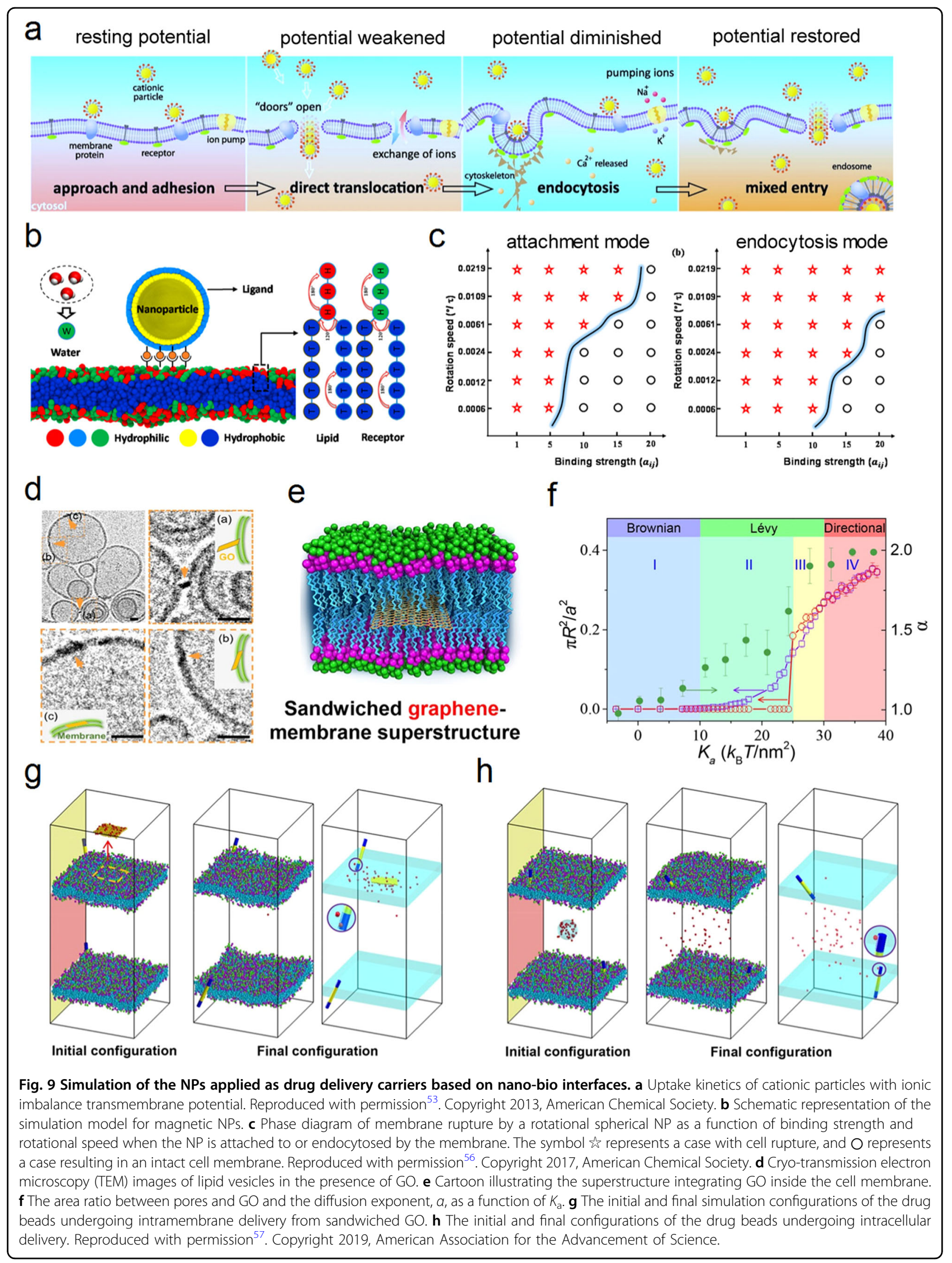


(Fig. 9d ${ }^{57}$. Chen et al. focused on the transport of ultrasmall GO sandwiched inside the cell membrane (Fig. 9e). The combined simulations and analysis identified a sandwiched GO-induced pore in cell membrane leaflets, which showed unstable, metastable, and stable states. An analytical model that rationalized the regimes of these membrane-pore states was used to fit the simulation results quantitatively, resulting in a mechanistic interpretation of the emergence of Lévy and directional dynamics (Fig. 9f). In principle, the findings of controllable pore states and resulting in persistent transport within cell membranes can affect the design of novel 2D nanocarriers for precision drug delivery (e.g., targeting membrane receptors) and unique therapeutic approaches. The applicability of the sandwiched GO to enhance the efficiency of membrane-specific drug delivery was demonstrated (Fig. 9g, h).

\section{Nanotoxicology}

The application of NPs in vivo inevitably disturbs the physiological functions of cells. Therefore, the nanotoxicity of NPs to cells must be considered. Specific effects can be determined by using cell and animal experiments; however, the underlying microscopic mechanism needs to be clarified using corresponding simulations. Lerner et al.$^{58}$ found that $\mathrm{Al}$ hydroxide nanosheets can inhibit tumor cell growth, and they employed a direct molecular dynamics simulation to analyze the interaction of nano-objects with biological structures. They revealed that $\mathrm{Al}$ hydroxide nanosheets can cause significant ion imbalance in the living cell perimembranous space through the selective adsorption of extracellular anionic species (e.g., $\mathrm{Cl}^{-}, \mathrm{HCO}^{-}$) (Fig. 10a-c). Such a reduction in extracellular $\mathrm{Cl}^{-}$concentration can inhibit cell growth, for example, by regulating the activity of ion channels and/or transporters ${ }^{59}$, which can be applied to explain the antiproliferative properties of Al hydroxide nanosheets. This novel mechanism can explain the killing effect on tumor cells caused by this type of NP.

Similarly, Tu et al..$^{60}$ adopted AAMD simulations to detect the cytotoxicity of pristine graphene nanosheets (GN) and GO. They found that both GN and GO nanosheets can induce the degradation of the inner and outer cell membranes and reduce cell viability. The simulations revealed that GN and GO nanosheets can penetrate into and extract large amounts of phospholipids from cell membranes because of the strong dispersion interactions between graphene and lipid molecules. This destructive extraction offered a novel mechanism for the a

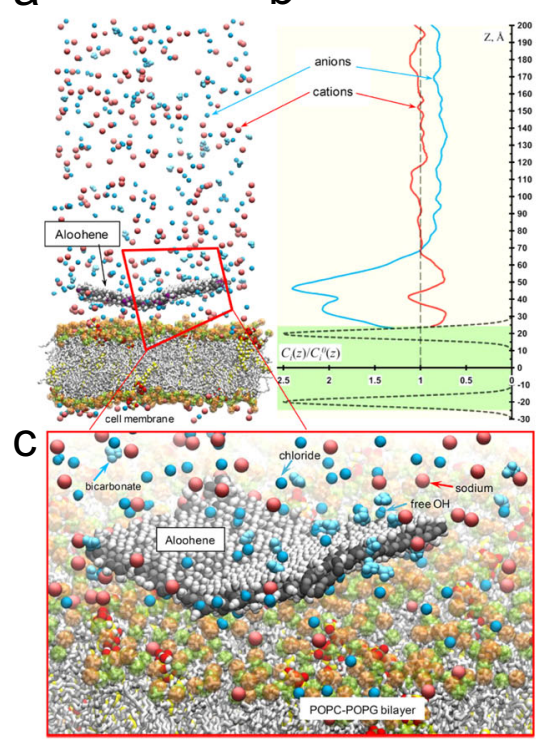

d
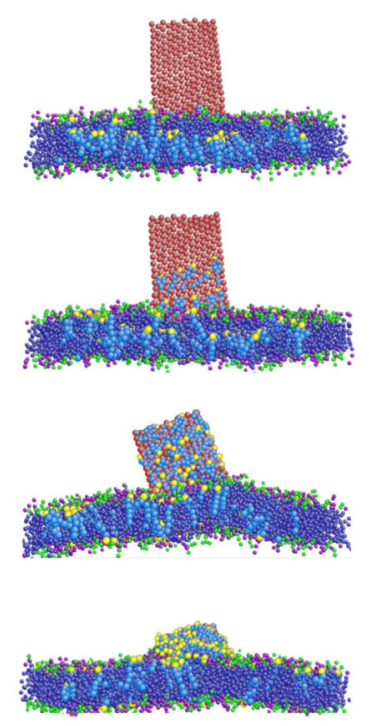

e

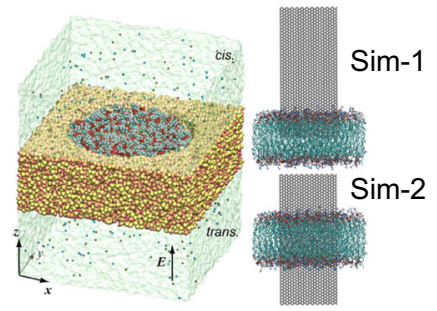

f

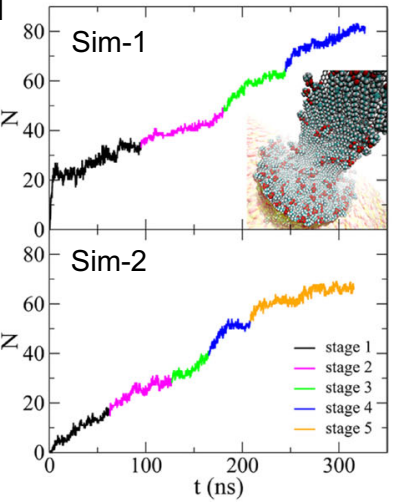

Fig. 10 Simulation of the toxicity of NPs based on nano-bio interfaces. a Spatial distribution of cations and anions in the extracellular area near the cell membrane in the presence of the Al hydroxide nanosheet. $\mathbf{b}$ lon concentration $C_{i}(z)$ of cations and anions relative to the unperturbed level for the same ion $C_{i}^{0}(z)$ at a distance $z$ from the cell membrane. $\mathbf{c}$ The insert demonstrates an enlarged fragment of the Al hydroxide nanosheet and the cell membrane. Reproduced with permission ${ }^{58}$. Copyright 2018, American Chemical Society. d Orientation of GN on the cell membrane. Reproduced with permission ${ }^{61}$. Copyright 2016, American Chemical Society. e Nanopore system with two modes: a graphene nanosheet enters halfway through the membrane (Sim-1), and a graphene nanosheet enters the membrane entirely with equally exposed segments on both sides of the membrane (Sim-2). f Time-dependent number of POPC lipids extracted onto the GN in Sim-1 and Sim-2. Reproduced with permission ${ }^{63}$. Copyright 2017, American Chemical Society. 
molecular basis of the cytotoxicity of GN and GO. Following this work, Zhang et al. ${ }^{61}$ expanded the cell membrane components from phospholipids to include cholesterol. They performed DPD simulations to investigate whether GN extracted cholesterol molecules from a cell membrane. They also demonstrated the capability of GN to absorb cholesterol molecules on its surface (Fig. 10d). The extraction of cholesterol molecules from the cell membrane can induce serious membrane curvature disruptions, which can potentially result in reduced cell viability. This allowed the assessment of the implications of GN in reducing the health risk associated with high cholesterol levels. Surface modification of GO is needed to reduce its cytotoxicity for practical applications. The protein corona has been reported as an effective method to mitigate the cytotoxicity of GO by reducing its physical interaction with the cell membrane ${ }^{62}$.

Additionally, some detection approaches using simulations can be utilized to detect the nanotoxicology of NPs based on ubiquitous nano-bio interfaces. For example, a nanopore-based single-molecule approach that can be applied to monitor a specific NP-membrane interaction in real-time has been developed (Fig. 10e). Combined with molecular dynamics and experimental approaches, Luan et al. ${ }^{63}$ showed how an ionic current can be used to detect real-time membrane damage using $\mathrm{GN}$ and illustrated the underlying molecular mechanism (Fig. 10f). The measured transmembrane ionic currents can be further developed and extended to achieve different particle-induced membrane modifications, such as hole formation, particle adsorption, and protein insertion.

\section{Protein function regulation}

When NPs interact with the cell membrane, they not only mediate disturbances in the cell membrane structure, such as lipid wrapping and membrane-pore formation, but also affect other components (mainly membrane proteins) of the membrane. Fluctuations in the transmembrane regions of membrane proteins are important because they are closely related to transmembrane signal transduction. Currently, the characterization of lipid-protein interactions and lipid-mediated modulation of membrane protein function have been explored using molecular simulations ${ }^{64,65}$. Significant attention has been given to the role of NPs in nano-bio interface interactions. $\mathrm{Hu}$ et $\mathrm{al}^{66}$, using CGMD simulation, focused on the mechanisms wherein inhaled NPs interacted with the pulmonary surfactant film and found that the surface charge of NPs regulated the formation of the pulmonary surfactant lipoprotein corona. It was observed that only anionic NPs (in blue) can selectively adsorb the SP- $B_{1-25}$ protein (not the SP-C protein) (purple). For the cationic and neutral NPs, although point contact was possible, SP$\mathrm{B}_{1-25}$ remained associated with the phospholipids without adsorbing onto the NP surfaces (Fig. 11a). A PMF analysis of the interaction between the surfactant monolayer and protein molecules explained the NP selectivity for SP$B_{1-25}$. The PMF of "pulling" an SP-C molecule was more than double that of "pulling" an SP- 1-25 $_{1-2}$ molecule, indicating a higher affinity of the surfactant monolayer with the SP-C molecule than with the SP- $\mathrm{B}_{1-25}$ molecule (Fig. 11b). These nano-bio interface interactions and the formation of lipoprotein coronas not only affect the biophysical properties of the pulmonary surfactant film but also play an important role in regulating subsequent biomolecular exchanges on NP surfaces, interactions with lung cells, and particle translocation to various organs and tissues.

Similarly, Luo et al. ${ }^{67}$ found that PEGylated GO (GOPEG) can stimulate potent cytokine responses in peritoneal macrophages despite not being internalized and activate the intracellular signaling pathway from integrin $\alpha_{v} \beta_{8}$ on the cell membrane (Fig. 11c). Corresponding AAMD simulations revealed a mechanism wherein GOPEG preferentially adsorbed onto and/or partially inserted into the cell membranes (Fig. 11d), thereby amplifying interactions with the stimulatory surface receptor integrin $\alpha_{v} \beta_{8}$. A recent simulation study further clarified the dynamic interaction process and detailed activation mechanism between GO-PEG and membrane protein $\alpha_{\mathrm{v}} \beta_{8}$ for feasible functional modification ${ }^{68}$. Moreover, Tian et al. ${ }^{69}$ investigated the effects of GO on actin filaments, whose physiological function is closely associated with cellular migration. AAMD simulations showed that GO nanosheets can be inserted into the interstrand gap of the actin tetramer (helical repeating unit of actin filament). This caused the separation of the tetramer, which eventually led to the disruption of the actin filaments.

In summary, it was observed that most of the present works model only binary systems comprising an NP with either a membrane or protein for AAMD simulation. This may be due to the complexity and irregularity of ternary systems, which require a large amount of computing resources, a deep understanding of the relationship between protein structure and function, and detailed data analysis. Although some CG models can be used to study ternary systems, they omit the veritable structural information of proteins, which greatly limits the reliability of the results. Thus, new ternary models should be developed and utilized to investigate nano-bio interface interactions.

\section{Conclusion}

Nano-bio interfaces between NPs and cell membranes occur widely in organisms. These nano-bio interfaces contain a series of time-dependent dynamic interactions, which dominate the physical and chemical interactions, kinetics, and thermodynamic exchanges between the NPs 


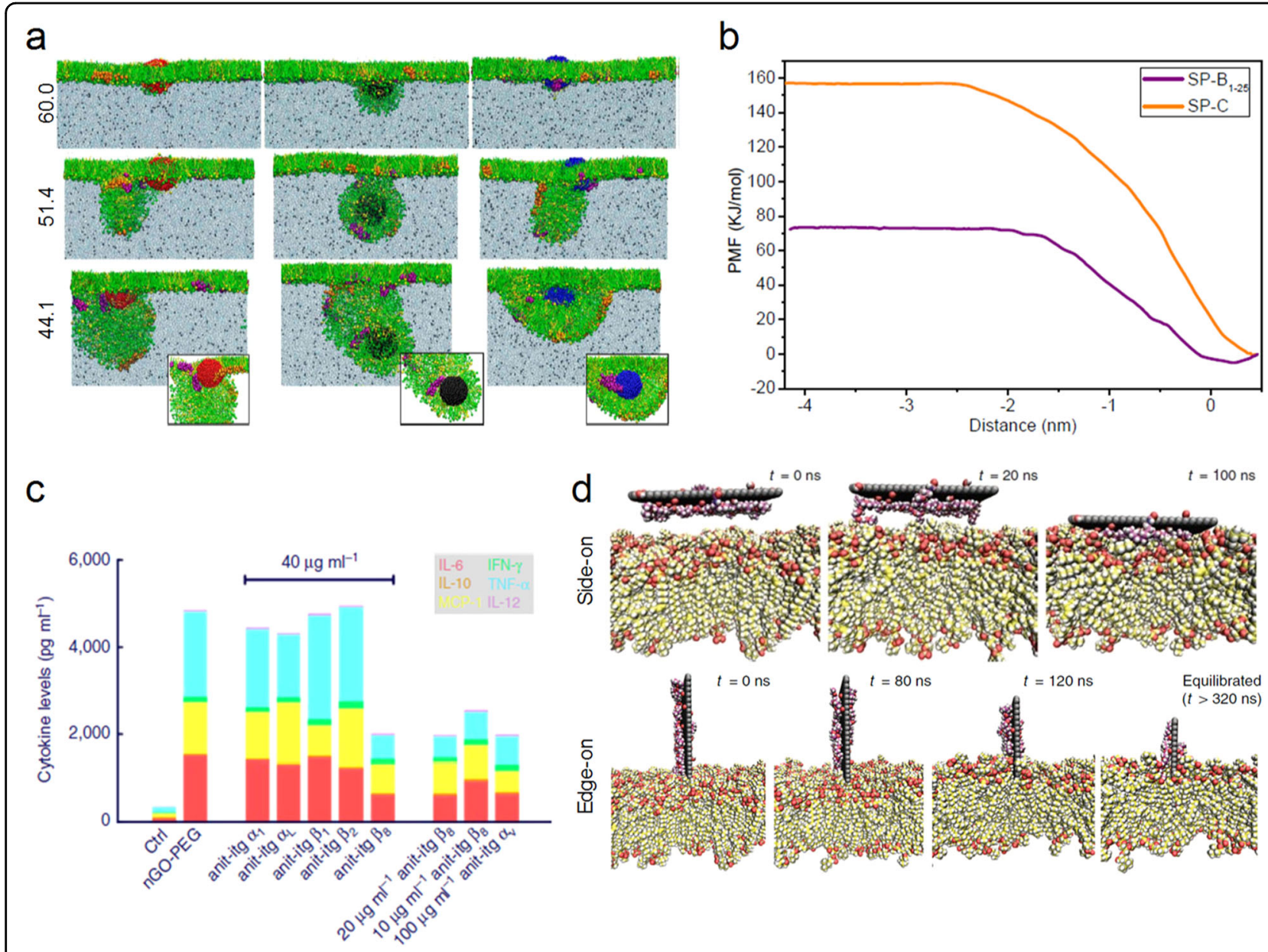

Fig. 11 Simulation of NPs regulating the function of the membrane protein based on nano-bio interfaces. a Computer-simulated interaction between a hydrophobic NP of different surface charges and a pulmonary surfactant film under increasing compression $\left(\AA^{2} / \mathrm{molecule}\right)$. b PMF for interaction between the surfactant monolayer and the protein molecules. Reproduced with permission ${ }^{66}$. Copyright 2013, American Chemical Society. c Secreted inflammatory cytokine concentrations after the application of different integrin inhibitors or $\beta_{8}$ siRNA interference. $\mathbf{d}$ Simulation of GO-PEG-membrane interactions from side-on and edge-on configurations. Reproduced with permission ${ }^{67}$. Copyright 2017, Springer Nature.

and cell membrane, hence stimulating cellular responses and mediating the corresponding physiological effects. NPs directly participate in these interface interactions; thus, their physiochemical properties (size, shape, elasticity, charge, hydrophobicity, and surface modification) are the dominant regulatory factors. Although it is clear that their effects on cellular uptake are strongly interrelated, a favorable physical property in principle can be summarized based on the simulations. This primary conclusion can reduce the design cost and time of NPs, provide explanations for experimental phenomena and guide potential biomedical applications.

This review focused entirely on simulations, with a detailed description and comprehensive comparison among the AAMD, CGMD, and DPD simulation methods. In fact, the gap between the simulations and experiments is significant in both temporal and spatial scales. The current simulation method makes it difficult to realize the spatiotemporal relationship of the experiment. Thus, new simulation methods and force potentials need to be developed. Specifically, multiscale simulation methods are promising for solving this problem ${ }^{70}$. A problem can be decomposed into several parts according to its spatial scale, covering magnitudes from nanometers to meters. Each part of this problem in each dimension can be explored using the appropriate model and method. By establishing suitable boundary conditions, the output of one part of the problem can be transmitted as the input of the neighboring part. Through a series of continuous transmissions, this problem can be clarified from the macro to the micro-scale, which makes the simulation results more consistent with physiological phenomena.

Considering the limitations of the simulation itself, the conclusions obtained by the simulation may not reflect the actual situation completely; therefore, they need to be further verified by corresponding experiments. In this 
paper, we systematically elucidated how the different physicochemical properties of NPs regulate nano-bio interfacial effects using simulations. Corresponding experiments are essential to verify the authenticity and reliability of the simulation conclusions. For example, NPs can be characterized using TEM (size and shape), atomic force microscopy (AFM) (elasticity, depth), zeta potential (charge), contact angle (hydrophobicity), and energydispersive X-ray spectroscopy (chemical modification). The mechanical parameters of nano-bio interface interactions can be determined using AFM, optical tweezers, or quartz crystal microbalance. The number of endocytosed NPs can be detected using confocal laser scanning microscopy imaging and flow cytometry analysis. In summary, a fundamental way to improve research efficiency is to predict experimental phenomena through simulations and verify simulation results through experiments to achieve an effective combination of simulations and experiments.

\section{Acknowledgements}

This work was supported by the National Natural Science Foundation of China (grant nos. 21821005, 32030062, and U2001224) and the Strategic Priority Research Program of the Chinese Academy of Sciences (XDB29040303).

\section{Competing interests}

The authors declare no competing interests.

\section{Publisher's note}

Springer Nature remains neutral with regard to jurisdictional claims in published maps and institutional affiliations.

Received: 26 February 2021 Revised: 28 May 2021 Accepted: 3 June 2021. Published online: 16 July 2021

\section{References}

1. Vert, M. et al. Terminology for biorelated polymers and applications (IUPAC Recommendations 2012). Pure Appl. Chem. 84, 377-410 (2012).

2. Batista, C. A., Larson, R. G. \& Kotov, N. A. Nonadditivity of nanoparticle interactions. Science 350, 1242477 (2015).

3. Tonga, G. Y., Saha, K. \& Rotello, V. M. 25th anniversary article: interfacing nanoparticles and biology: new strategies for biomedicine. Adv. Mater. 26, 359-370 (2014).

4. Panwar, N. et al. Nanocarbons for biology and medicine: sensing, imaging, and drug delivery. Chem. Rev. 119, 9559-9656 (2019).

5. Lin, H., Chen, Y. \& Shi, J. Nanoparticle-triggered in situ catalytic chemical reactions for tumour-specific therapy. Chem. Soc. Rev. 47, 1938-1958 (2018).

6. Wang, Y., Cai, R. \& Chen, C. The nano-bio interactions of nanomedicines: understanding the biochemical driving forces and redox reactions. Acc. Chem. Res. 52, 1507-1518 (2019).

7. Nel, A. E. et al. Understanding biophysicochemical interactions at the nano-bio interface. Nat. Mater. 8, 543-557 (2009).

8. Donahue, N. D., Acar, H. \& Wilhelm, S. Concepts of nanoparticle cellular uptake, intracellular trafficking, and kinetics in nanomedicine. Adv. Drug Deliv. Rev. 143 68-96 (2019)

9. Gagner, J. E., Shrivastava, S., Qian, X., Dordick, J. S. \& Siegel, R. W. Engineering nanomaterials for biomedical applications requires understanding the nanobio interface: a perspective. J. Phys. Chem. Lett. 3, 3149-3158 (2012).

10. Karplus, M. \& Petsko, G. A. Molecular dynamics simulations in biology. Nature 347, 631-639 (1990)
11. Charchar, P., Christofferson, A. J., Todorova, N. \& Yarovsky, I. Understanding and designing the gold-bio interface: insights from simulations. Small 12, 2395-2418 (2016).

12. Moeendarbary, E. Ng, T. Y. \& Zangeneh, M. Dissipative particle dynamics: introduction, methodology and complex fluid applications-a review. Int. J. Appl. Mech. 1, 737-763 (2012).

13. Alberts, B. et al. The Lipid Bilayer - Molecular Biology Of The Cell 4th edn (Garland Science, New York, 2002).

14. Henry, E. R., Best, R. B. \& Eaton, W. A. Comparing a simple theoretical model for protein folding with all-atom molecular dynamics simulations. Proc. Natl Acad. Sci. USA 110, 17880-17885 (2013).

15. Klauda, J. B. et al. Update of the CHARMM all-atom additive force field for lipids: validation on six lipid types. J. Phys. Chem. B 114, 7830-7843 (2010).

16. Takada, S. et al. Modeling structural dynamics of biomolecular complexes by coarse-grained molecular simulations. Acc. Chem. Res. 48, 3026-3035 (2015).

17. Marrink, S. J., Risselada, H. J., Yefimov, S., Tieleman, D. P. \& de Vries, A. H. The MARTINI force field: coarse grained model for biomolecular simulations. J. Phys. Chem. B 111, 7812-7824 (2007).

18. Souza, P. C. T. et al. Martini 3: a general purpose force field for coarse-grained molecular dynamics. Nat. Methods 18, 382-388 (2021).

19. Shi, X. \& Tian, F. Multiscale modeling and simulation of nano-carriers delivery through biological barriers-a review. Adv. Theor. Simul. 2 1800105 (2018).

20. Reif, M. M., Winger, M. \& Oostenbrink, C. Testing of the GROMOS force-field parameter set 54A8: structural properties of electrolyte solutions, lipid bilayers, and proteins. J. Chem. Theory Comput. 9, 1247-1264 (2013).

21. Siu, S. W. Pluhackova, K. \& Bockmann, R. A. Optimization of the OPLS-AA force field for long hydrocarbons. J. Chem. Theory Comput. 8, 1459-1470 (2012).

22. Dickson, C. J. et al. Lipid14: the Amber lipid force field. J. Chem. Theory Comput. 10, 865-879 (2014).

23. Behzadi, S. et al. Cellular uptake of nanoparticles: journey inside the cell. Chem Soc. Rev. 46, 4218-4244 (2017).

24. Zhang, S., Gao, H. \& Bao, G. Physical principles of nanoparticle cellular endocytosis. ACS Nano 9, 8655-8671 (2015).

25. Huang, C., Zhang, Y., Yuan, H., Gao, H. \& Zhang, S. Role of nanoparticle geometry in endocytosis: laying down to stand up. Nano Lett. 13, 4546-4550 (2013).

26. Gupta, R. \& Rai, B. Effect of size and surface charge of gold nanoparticles on their skin permeability: a molecular dynamics study. Sci. Rep. 7, 45292 (2017).

27. Yue, T. \& Zhang, X. Cooperative effect in receptor-mediated endocytosis of multiple nanoparticles. ACS Nano 6, 3196-3205 (2012).

28. Guo, R., Mao, J. \& Yan, L. T. Computer simulation of cell entry of graphene nanosheet. Biomaterials 34, 4296-4301 (2013).

29. Yang, K. \& Ma, Y. O. Computer simulation of the translocation of nanoparticles with different shapes across a lipid bilayer. Nat. Nanotechnol. 5, 579-583 (2010).

30. Gupta, R., Badhe, Y., Mitragotri, S. \& Rai, B. Permeation of nanoparticles across the intestinal lipid membrane: dependence on shape and surface chemistry studied through molecular simulations. Nanoscale $\mathbf{1 2}$ 6318-6333 (2020).

31. Shen, Z., Ye, H., Yi, X. \& Li, Y. Membrane wrapping efficiency of elastic nanoparticles during endocytosis: size and shape matter. ACS Nano 13, 215-228 (2019).

32. Vacha, R., Martinez-Veracoechea, F. J. \& Frenkel, D. Receptor-mediated endocytosis of nanoparticles of various shapes. Nano Lett. 11, 5391-5395 (2011).

33. Li, Y., Yue, T., Yang, K. \& Zhang, X. Molecular modeling of the relationship between nanoparticle shape anisotropy and endocytosis kinetics. Biomaterials 33, 4965-4973 (2012).

34. Sun, J. et al. Tunable rigidity of (polymeric core)-(lipid shell) nanoparticles for regulated cellular uptake. Adv. Mater. 27, 1402-1407 (2015).

35. Shen, Z., Ye, H. \& Li, Y. Understanding receptor-mediated endocytosis of elastic nanoparticles through coarse grained molecular dynamic simulation. Phys. Chem. Chem. Phys. 20, 16372-16385 (2018).

36. Shen, Z., Ye, H., Kröger, M. \& Li, Y. Aggregation of polyethylene glycol polymers suppresses receptor-mediated endocytosis of PEGylated liposomes. Nanoscale 10, 4545-4560 (2018).

37. Nangia, S. \& Sureshkumar, R. Effects of nanoparticle charge and shape anisotropy on translocation through cell membranes. Langmuir 28, 17666-17671 (2012). 
38. Lin, X., Wang, C., Wang, M., Fang, K. \& Gu, N. Computer simulation of the effects of nanoparticles' adsorption on the properties of supported lipid bilayer. J. Phys. Chem. C 116, 17960-17968 (2012).

39. Li, Y. \& Gu, N. Thermodynamics of charged nanoparticle adsorption on charge-neutral membranes: a simulation study. J. Phys. Chem. B 114, 2749-2754 (2010).

40. Marcano, D. C. et al. Improved synthesis of graphene oxide. ACS Nano 4, 4806-4814 (2010).

41. Gupta, R. \& Rai, B. In-silico design of nanoparticles for transdermal drug delivery application. Nanoscale 10, 4940-4951 (2018).

42. Su, C. F., Merlitz, H., Rabbel, H. \& Sommer, J. U. Nanoparticles of various degrees of hydrophobicity interacting with lipid membranes. J. Phys. Chem. Lett. 8, 4069-4076 (2017).

43. Sedlmeier, A. \& Gorris, H. H. Surface modification and characterization of photon-upconverting nanoparticles for bioanalytical applications. Chem. Soc. Rev. 44, 1526-1560 (2015).

44. Kankala, R. K. et al. Nanoarchitectured structure and surface biofunctionality of mesoporous silica nanoparticles. Adv. Mater. 32, e1907035 (2020).

45. Khutoryanskiy, V. V. Beyond PEGylation: alternative surface-modification of nanoparticles with mucus-inert biomaterials. Adv. Drug Deliv. Rev. 124, 140-149 (2018).

46. Shen, Z., Baker, W., Ye, H. \& Li, Y. pH-Dependent aggregation and pHindependent cell membrane adhesion of monolayer-protected mixed charged gold nanoparticles. Nanoscale 11, 7371-7385 (2019).

47. Van Lehn, R. C. et al. Effect of particle diameter and surface composition on the spontaneous fusion of monolayer-protected gold nanoparticles with lipid bilayers. Nano Lett. 13, 4060-4067 (2013).

48. Bai, X., Xu, M., Liu, S. \& Hu, G. Computational investigations of the interaction between the cell membrane and nanoparticles coated with a pulmonary surfactant. ACS Appl. Mater. Interfaces 10, 20368-20376 (2018).

49. Bao, W. et al. Experimental and theoretical explorations of nanocarriers' multistep delivery performance for rational design and anticancer prediction. Sci. Adv. 7, eaba2458 (2021).

50. Chen, P., Xu, Z., Zhu, G., Dai, X. \& Yan, L. T. Cellular uptake of active particles. Phys. Rev. Lett. 124, 198102 (2020).

51. Xu, Z., Gao, L., Chen, P. \& Yan, L. T. Diffusive transport of nanoscale objects through cell membranes: a computational perspective. Soft Matter 16, 3869-3881 (2020).

52. Deng, H., Dutta, P. \& Liu, J. Stochastic modeling of nanoparticle internalization and expulsion through receptor-mediated transcytosis. Nanoscale 11, 11227-11235 (2019).

53. Lin, J. \& Alexander-Katz, A. Cell membranes open "doors" for cationic nanoparticles/biomolecules: insights into uptake kinetics. ACS Nano 7, 10799-10808 (2013).
54. Shimizu, K., Nakamura, H. \& Watano, S. MD simulation study of direct permeation of a nanoparticle across the cell membrane under an external electric field. Nanoscale 8, 11897-11906 (2016).

55. Zhang, L. \& Wang, X. Coarse-grained modeling of vesicle responses to active rotational nanoparticles. Nanoscale 7, 13458-13467 (2015).

56. Zhang, L., Zhao, Y. \& Wang, X. Nanoparticle-mediated mechanical destruction of cell membranes: a coarse-grained molecular dynamics study. ACS Appl. Mater. Interfaces 9, 26665-26673 (2017).

57. Chen, P. et al. Transport of a graphene nanosheet sandwiched inside cell membranes. Sci. Adv. 5, eaaw3192 (2019).

58. Lerner, M. I. et al. Crumpled aluminum hydroxide nanostructures as a microenvironment dysregulation agent for cancer treatment. Nano Lett. 18, 5401-5410 (2018)

59. Collier, D. M. \& Snyder, P. M. Extracellular chloride regulates the epithelial sodium channel. J. Biol. Chem. 284, 29320-29325 (2009).

60. Tu, Y. et al. Destructive extraction of phospholipids from Escherichia coli membranes by graphene nanosheets. Nat. Nanotechnol. 8, 594-601 (2013).

61. Zhang, L., Xu, B. \& Wang, X. Cholesterol extraction from cell membrane by graphene nanosheets: a computational study. J. Phys. Chem. B 120, 957-964 (2016).

62. Duan, G. et al. Protein corona mitigates the cytotoxicity of graphene oxide by reducing its physical interaction with cell membrane. Nanoscale 7, 15214-15224 (2015).

63. Luan, B., Zhou, S., Wang, D. \& Zhou, R. Detecting interactions between nanomaterials and cell membranes by synthetic nanopores. ACS Nano 11, 12615-12623 (2017)

64. Muller, M. P. et al. Characterization of lipid-protein interactions and lipidmediated modulation of membrane protein function through molecular simulation. Chem. Rev. 119, 6086-6161 (2019).

65. Goossens, K. \& De Winter, H. Molecular dynamics simulations of membrane proteins: an overview. J. Chem. Inf. Model. 58, 2193-2202 (2018).

66. Hu, G. et al. Physicochemical properties of nanoparticles regulate translocation across pulmonary surfactant monolayer and formation of lipoprotein corona. ACS Nano 7, 10525-10533 (2013).

67. Luo, N. et al. PEGylated graphene oxide elicits strong immunological responses despite surface passivation. Nat. Commun. 8, 14537 (2017).

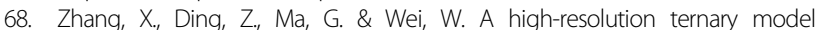
demonstrates how PEGylated 2D nanomaterial stimulates integrin $a_{v} \beta_{8}$ on cell membrane. Adv. Sci. 8, 2004506 (2021).

69. Tian, $X$. et al. Graphene oxide nanosheets retard cellular migration via disruption of actin cytoskeleton. Small 13, 1602133 (2017).

70. Eckmann, D. M. et al. Multiscale modeling of protein membrane interactions for nanoparticle targeting in drug delivery. Curr. Opin. Struc. Biol. 64, 104-110 (2020). 